\title{
Europasoziologie im Querschnitt
}

\author{
Buchbesprechung zu: Maurizio Bach \& Barbara Hönig. \\ Europasoziologie. Handbuch für Wissenschaft und Studium. \\ Baden Baden: Nomos, 2018
}

\author{
Martin Seeliger (martin.seeliger@uni-flensburg.de) \\ Europa-Universität Flensburg, Germany
}

\begin{abstract}
„Mit dem Auf- und Ausbau der EU ist ein einzigartiges zeitgeschichtliches Laboratorium für die soziologische Beobachtung von Prozessen der Institutionenbildung und ihren gesamtgesellschaftlichen Konsequenzen entstanden“ (Bach \& Hönig, 10). Gut ein Vierteljahrhundert nach dem Beschluss der Maastrichter Verträge könnte die Begründung, zu welchem Ende man Europasoziologie betreiben sollte, wohl kaum weniger attraktiv und spannend ausfallen. Die über 50 Beiträge des Handbuches Europasoziologie (herausgegeben von Maurizio Bach und Barbara Hönig) sind in sechs Abschnitte gegliedert, „entlang derer“, so begründen die beiden ihre Zusammenstellung, „sich die Forschung in den vergangenen zweieinhalb Jahrzehnten ihr Profil gewonnen hat" (Bach \& Hönig, 10). Anschließend an eine Vorstellung des Themas umfasst die typische Gliederung die Darstellung des Literaturstands unter jeweils individuell prononcierten Aspekten und schließlich die Vorstellung weiterer konkreter Forschungsfragen und allgemeiner Desiderate.
\end{abstract}

Unter dem Titel ,Soziologische Kartografien Europas'versammelt der erste Abschnitt eine Reihe makrosoziologischer Perspektiven und Reflexionen über Grundbegriffe soziologischen Denkens auf und über Europa. „Die europäische Gesellschaft" lässt sich mit Hans-Peter Müller (44) „als eine Konfiguration von kulturellen Traditionen und Werten, historischen Erfahrungen und spezifisch ausgestalteten Institutionen und ihrer Ordnung fassen." Ein zentrales Merkmal, welches die Autoren aus diesem Teil des Buches der europäischen Gesellschaft zuschreiben, ist ihre Uneindeutigkeit: Sie sei weder geplant, noch chaotisch, weder latent, noch manifest. Während die EU einerseits, so Vobruba (52) „die Avantgarde unter den vielfaltigen politisch-ökonomischen Ansätzen zur Entwicklung postnationaler Räume“ und mit ihren Regierungsorganisationen eine „politischadministrative Institution" darstellt, "die in der Geschichte gänzlich ohne Vorbild ist" (Bach, 60), fehlt ihrem politischen System gleichzeitig eine eindeutige Struktur der Verantwortlichkeiten. Der gesellschaftstheoretische Anspruch in diesem Teil des Buches wird durch eine umfassende Berücksichtigung kultur-, politik- und wirtschaftssoziologischer Aspekte eingelöst.

In einem zweiten Abschnitt zur, Institutionenbildung und Institutionenpolitik' widmet sich eine zweite Gruppe von Beiträgen der Darstellung von Strukturprinzipien und Verlaufsmustern institutioneller Logiken und Prozesse auf europäischer Ebene. Über die Darstellung einer gemeinschaftlichen Arbeitsmarkt- und Beschäftigungspolitik und die Bürokratisierung der EU reichen die Themen hier bis zum Diskriminierungsverbot, dem Europäischen Forschungsraum oder der Integration durch Recht. Die Abgrenzung der Felder erfolgt häufig relativ trennscharf, so ziehen z.B. Schmidt-Wellenburg und Mackert anschließend an T.H. Marshall mit der Evolution von Staatsbürgerrechten zwar die Ent- 
wicklung von Ansprüchen gegenüber dem Gemeinwesen in Betracht - inwiefern sich dies z.B. in die tarifpolitische Arena übertragt (Marshall bezeichnet dies als, Industrial Citizenship'), bleibt hier unerwähnt. Ein gemeinsames Motiv der Beiträge findet sich auch hier in dem Verweis auf die Tatsache, dass die EU zwar durch Elemente von Staatlichkeit charakterisiert, jedoch deswegen noch lange kein politisches System im klassischnationalstaatlichen Sinne darstelle.

Die Frage nach der territorialen Verfasstheit Europas stellt sich vor allem vor dem Hintergrund der Erweiterungsrunden der Jahre 2004 und 2007, welche den Einfluss der EU im räumlichen Maßstab weiter erhöhen konnten, aber auch ihre interne Heterogenität weiter intensiviert haben. Konkret geht es hier um Grenzräume in Europa, sowie Grenzsicherungspolitik, Integration und Erweiterung der Union, europäische Nachbarschaftspolitik sowie die Transformation Ostmittel- und Südosteuropas.

Die Beiträge zum folgenden Abschnitt ,Sozialstruktur und Sozialpolitik' thematisieren Möglichkeiten und Grenzen der Organisation und Wirkungsweise marktbegrenzender Institutionen. Das Spannungsverhältnis ergibt sich hierbei zum einen aus der wachsenden Menge und Heterogenität der Teilnehmer am gemeinsamen Markt sowie zum anderen aus der Kompetenzverschiebung in der Vertikalen des EU-Mehrebenensystems. Eine dritte wesentliche Rahmenbedingung stellt zudem die Eurokrise sowie die hieraus resultierende Austeritätspolitik dar. Über theoretische Reflexionen zur Europäisierung sozialer Ungleichheit reichen die inhaltlichen Schwerpunkte in diesem Abschnitt über die Europäisierung industrieller Beziehungen und Migration bis hin zur Thematisierung politischer Konflikte um soziale Ungleichheit. Aus der Tatsache, dass sich „das Terrain der Sozialstruktur Europas [als] unübersichtlich“ (Mau \& Verwiebe, 270) darstellt, ergibt sich damit für diesen Bereich ein besonderer methodologischer Forschungsbedarf. Wie sollen wir die Sozialstruktur einer Gesellschaft untersuchen, welche sich immer weniger nationalstaatlich segmentiert? Und wie ließen sich entsprechende Befunde auf die Analyse von Wirtschaft und Gesellschaft Europas im internationalen und globalen Kontext übertragen?

Auf ,Transnationale soziale Prozesse' innerhalb Europas heben die Beiträge des folgenden Abschnittes ab. Im Sinne einer transnationalen Forschungsperspektive geht es hier in erster Linie um lebensweltlich rückgebundene Sequenzen sozialen Handelns im grenzüberschreitenden Maßstab, welche in unterschiedlichen gesellschaftlichen Bereichen in Zusammenhang mit der europäischen Einigung stehen. Kollektive Erinnerungsprozesse werden hier genauso thematisiert wie die Eurokrise oder die Dynamiken und Bedeutung euroskeptischer Haltungen, Generationenbeziehungen in Migrantenfamilien oder die Frage nach einer europäischen Öffentlichkeit. Um dies gleich vorwegzunehmen - die Vielfalt der Gegenstände ist keineswegs rein willkürlich begründet, sondern bildet die Multidimensionalität europasoziologisch relevanter Gegenstände ab.

Der sechste Teil fasst ,Gesellschaftstheoretische Perspektiven' der Europasoziologie zusammen, indem er Beiträge aus den Bereichen der Feldtheorie, der Gouvernmentalitätstheorie, der Arbeiten zur Informationsgesellschaft, des historischen und des NeoInstitutionalismus, sowie Fragen nach der Transformation gesellschaftlicher Solidarität auf den Gegenstandsbereich der Europasoziologie bezieht. Wenn man davon ausgeht, dass eine Theorie diejenigen Aspekte bestimmt, unter denen ein spezifischer Forschungsgegenstand definiert und untersucht wird, zeigen die Beiträge in diesem Bereich auf anschauliche Weise die Komplexität des europäischen Einigungsprozesses. Ganz in diesem Sinne problematisieren die Beiträge zum letzten Abschnitt schließlich ,For- 
schungsstrategien und Methodenkritik' mit Blick auf Design und Operationalisierung europasoziologischer Forschung. Besonders interessant (und sicherlich diskussionswürdig) erscheint hier der Beitrag von Pickel, der Limitationen und affektuelle Probleme feldspezifischer Fachvertreter im Umgang mit quantitativen Methoden moniert.

Insgesamt legen die HerausgeberInnen und AutorInnen mit dem Handbuch ein (wenn nicht das) Referenzwerk vor, das über die (ohnehin unklar verlaufenden) Grenzen der Europasoziologie hinaus den Forschungsdiskurs der nächsten Jahre in vielerlei Hinsicht beeinflussen wird. Die Beiträge beleuchten den Forschungsstand in den dargestellten Feldern hierbei in unterschiedlicher Tiefe und setzen die Pointen in unterschiedlich starker Intensität. Auch die Vorschläge für zukünftige Forschungsgegenstände fallen mal mehr und mal weniger klar aus und die Verbindung zwischen den jeweils behandelten Grundbegriffen und dem empirisch zu erschließenden Gegenstandsbereich weisen insgesamt eine variierende Qualität auf. Dies zeigt sich nicht zuletzt beim Durchgehen der Literaturverzeichnisse der jeweiligen Beiträge - während sich einige besonders umfangreiche und sorgfältig zusammengestellt prinzipiell auch als Review-Artikel lesen lassen, wirken andere hier eher kursorisch und tendenziös (das ist schade, denn in der sorgfältigen Rekonstruktion und Abbildung des Literaturstandes kann ja einer der ganz wesentlichen Beiträge eines solchen Textes liegen).

Es ließe sich außerdem über die Auswahl der Themenfelder diskutieren. Wieso gibt es keinen eigenen Beitrag zu den vier Marktfreiheiten und insbesondere den Einfluss der Dienstleistungsfreiheit auf die Funktionsweise nationaler Arbeitsmärkte? Gibt es eigentlich so etwas wie eine europäische Populärkultur? Welche Rolle spielen multinationale Unternehmen im Prozess der Europäischen Integration? Und was ist mit der neuen Arbeiterbewegung von rechts, deren Erstarken wir nicht zuletzt vor dem Hintergrund der Eurokrise beobachten können? Streiten ließe sich auch darüber, ob man wirklich einen Beitrag über Migration in Mehrgenerationenfamilien braucht, oder nicht lieber stattdessen über die Kolonialvergangenheit Europas oder die Frage nach einer gemeinsamen Sicherheitspolitik der EU reflektieren sollte (usw., usf.). Dass die Zusammenstellung der Beiträge ab einem gewissen Punkt willkürlich und unvollständig sein muss, unterstreicht einmal mehr die Vielfalt und Komplexität der Europasoziologie genauso wie die Tatsache, dass soziologische Arbeiten über Europa sich mit einem ,moving target' befassen. ${ }^{1}$

Die Programmatik der, wie die Herausgeber erklären, seit zwanzig Jahren betriebenen Disziplin markiert „die pragmatische Bezeichnung Europasoziologie“ (Bach \& Hönig, 10): „Nicht theoretische Dogmatik, sondern multi-paradigmatische Suchbewegungen charakterisieren die neue Forschungsrichtung, bei der es sich immer noch eher um ein wissenschaftliches Experimentierfeld als um eine intellektuelle "Schule' handelt." Das klingt nicht nur spannend, sondern auch sympathisch und wirft schließlich die (evtl. sogar rhetorische?) Frage auf, in welchem anderen Bereich der Soziologie so grundsätzliche Probleme auf so vielfältige Weise und im Dialog unterschiedlicher Provenienzen bearbeitet werden.

\footnotetext{
${ }^{1}$ Bemerkenswerterweise betont auch Immerfall $(2018,3)$, dass sich die Aufgabe einer Einführung in die Soziologie Europas „von fortbestehenden Datenlücken und Theoriemangeln ganz abgesehen - [...] ohnehin nur in einem Gemeinschaftswerk bewältigen“ ließe. Nichts anderes ist im Falle des vorliegenden Handbuches geschehen.
} 
Leider ist dies aber nur die eine Seite. Denn während die Zusammenstellung der Beiträge zur Europasoziologie (bzw. zum Handbuch Europasoziologie) einerseits dem Prinzip einer programmatischen Offenheit folgt, weisen die Beiträge (sowohl in ihrer Zusammenstellung als auch in ihrem jeweiligen Argumentationsgang) einige Begrenzungen auf, die in verschiedenerlei Hinsicht als erklärungsbedürftig erscheinen. Während sowohl Herausgeber als auch die Autoren an verschiedener Stelle in Bezug auf das Ziel einer umfassenden Verständnis genuin europäischer Phänomene auf die Notwendigkeit einer gezielten Überschreitung (sub-)disziplinärer Grenzen (z.B. zwischen Soziologie und Politikwissenschaft) verweisen, bleibt dieser Wunsch im Rahmen der Beiträge häufig unerfüllt und es erscheint auch fraglich, inwiefern die sechs Abschnitte tatsächlich diejenigen Differenzierungslinien abbilden, „entlang derer sich die Forschung in den vergangenen zweieinhalb Jahrzehnten ihr Profil gewonnen hat" (Bach \& Hönig, 10).

Dies zeigt sich, so möchte ich argumentieren, (zumindest exemplarisch) an der weitgehenden Vernachlässigung politökonomischer Literaturstränge. In erster Linie betrifft dies Studien aus dem Bereich der Vergleichenden Politischen Ökonomie, welche die institutionellen Gegebenheiten in den europäischen Ländern zum Gegenstand hat. So lesen wir etwa im Artikel zur Eurokrise wenig über die Konstruktionsmängel der Währungsregimes, welche nicht zuletzt auf die ungleiche Entwicklung der nominalen Lohnstückkosten in den jeweils variierenden nationalen Produktionssystemen zurückzuführen sind (vgl. Höpner \& Lutter, 2014). Insgesamt scheinen die arbeits- und sozialpolitisch orientierten Beiträge der Persistenz nationaler Interessenlagen nicht in umfangreichen Maße Rechnung zu tragen. Dieser Leerstelle steht im selben Beitrag dann jedoch eine bemerkenswerte Institutionentreue gegenüber, welche sich offenbart, wenn Bach (293) mit Blick auf die Eurokrise bemerkt, dass die Mitgliedstaaten „in gleichem Maße und unauflöslich an die gemeinsame Währung gefesselt" seien. ${ }^{2}$ Dem Austritt einzelner Länder aus dem (oder sogar der Abschaffung des) Euro mag man nun politisch gegenüberstehen, wie man möchte. Ihre Existenz zu reifizieren entspricht jedoch nicht dem Primat ontologischer Skepsis, welches etwa Norbert Elias (2009) in Bezug auf eine soziologische Agenda der "Mythenjagd“ proklamiert. ${ }^{3}$

Ein Stück weit ist es natürlich normal, dass die Zusammenstellung eines Bandes immer auch das professionelle Umfeld der HerausgeberInnen abbildet. Das (v.a. im deutschsprachigen Raum verfolgte) Projekt der ,Europasoziologie' scheint hierbei stark an den personellen Rahmen der gleichnamigen Sektion aus der Deutschen Gesellschaft für Soziologie gebunden. Es wäre zu diskutieren, ob eine Weiterentwicklung der Disziplin nicht auch die gezielte Überschreitung oder sogar Einebnung auf beiden Seiten relevanter „Wasserscheiden der Wahrnehmung" (Beck, 2008, 11) erfordert. Als mögliches Vorbild

\footnotetext{
2 Die adäquate Referenzliteratur wäre hier vermutlich auch eher Scharpf (2012; 2012a oder 2014) als Beck (2005), wie bei Bach (299).

${ }^{3}$ Wenn der Euro scheitert, scheitert - anders als Angela Merkel dies einmal verlauten ließ - nämlich nicht Europa. Europa ist ein Kontinent und kann gar nicht scheitern, weil es als solcher keinem höheren Zweck dient. Was hier eventuell scheitern könnte, wäre die EU als politisches System, aber auch hierüber wäre wenigstens zu diskutieren. Dies wird jedoch erschwert, wenn jemand von vornherein eine ,Untrennbarkeit' postuliert. Würde man einen reflexiven Beitrag zur Europasoziologie anstreben, so gälte es - ganz im epistemologiekritischen Sinne Ulrich Becks - weiterhin ihre Fixierung auf ein spezifisches Ideal sozialer Gerechtigkeit in den Blick zu nehmen, welches neben der Reduktion sozialer Ungleichheit unter den Menschen („Ein regulierter Kapitalismus ist immer noch besser als ein neoliberaler. Über Enteignung und Planwirtschaft reden wir nicht, oder zumindest weder gern noch positiv.") auch die Privilegierung einer Idee kosmopolitischer Gesellschaftssteuerung als Notwendigkeit wahrhaftig demokratischer Ordnung impliziert („Wir brauchen starke supranationale Institutionen zur Regulierung internationaler Märkte!“). Nicht dass es mit irgendeiner der genannten Positionen ein grundsätzliches Problem geben muss - man sollte eben nur bewusst machen, unter welchen Prämissen man da operiert.
} 
könnte hier etwa der dezidiert interdisziplinär orientierte ,Council for European Studies' dienen. Und letztlich stellt sich natürlich auch die Frage, inwiefern eine Beschränkung der Teilnehmerschaft auf den deutschsprachigen Raum dem multi- und transnationalen Charakter des Gegenstandes ,Europa' gerecht wird.

Aber wie dem auch sei - das Handbuch Europasoziologie ist ein mehr als gelungener Band, der sorgfältig und klug aus insgesamt sehr gelungenen Beiträgen zusammengestellt ist, und so den Zugang zu einem spannenden und vielversprechenden Forschungsfeld eröffnet!

\section{Literatur}

Beck, U. (2005). Europäisierung - Soziologie für das 21. Jahrhundert. Aus Politik und Zeitgeschichte, 52(34-35), 3-10.

Beck, U. (2011). Die Neuvermessung der Ungleichheit unter den Menschen. Soziologische Aufklärung im 21. Jahrhundert - Eröffnungsvortrag zum Soziologentag "Unsichere Zeiten" am 6. Oktober 2008 in Jena. Frankfurt a.M.: Suhrkamp.

Elias, N. (2009). Was ist Soziologie? Grundfragen der Soziologie. Weinheim: Juventa.

Höpner, M. (2011). Der europäische Gerichtshof als Motor der europäischen Integration: Eine akteursbezogene Erklärung. Berliner Journal für Soziologie 21(2), 203-229.

Immerfall, S. (2018). Europa - Politisches Einigungswerk und gesellschaftliche Entwicklung. Wiesbaden: Springer.

Scharpf, F. W. (2012a). Legitimacy intermediation in the multilevel European polity and its collapse in the Euro crisis (MPIfG Discussion Paper No. 12/6.).

Scharpf, F. W. (2012b). Was soll und kann die Europäische Union? Zeitschrift für Staatsund Europawissenschaften 10(4), 540-550.

Scharpf, F. W. (2014). No exit from the Euro-Rescuing trap? (MPIfG Discussion Paper No. 14/4). 